\title{
Acotación heterotópica de la migración internacional: El valor espacial del patrimonio geográfico
}

\author{
Miriam Reyes Tovar ${ }^{1}$ \\ Isaías Daniel Hinojosa Flores²
}

\begin{abstract}
Resumen: El presente trabajo retoma el concepto de heterotopía realizado por el filósofo francés Michael Foucault, para virar su reflexión hacia uno de los fenómenos geográficos más interesantes respecto a la construcción de sentido de habitar, apropiar y significar territorios y lugares: la migración internacional. En este marco de comprensión, la construcción hacia esos "otros espacios" situadas en lo que, denominamos espacio real, en alusión al sociólogo y filosofo francés Henri Lefebvre, permite a este trabajo, postular la importancia que posee la vivencialidad y visibilidad de las diferencias en la comprensión del patrimonio geográfico y cultural de los encuentros, esto como una forma de observar la riqueza que posee la pluralidad y como una forma de resistencia a lo estipulado hegemónicamente ante el entender la diversidad.
\end{abstract}

Palabras clave: Migración internacional, heterotopía, espacio social, patrimonio geográfico, patrimonio cultural

\section{Delimitação heterotópica da migração internacional: O valor do patrimônio geográfico}

Resumo: O presente trabalho aborda o conceito de heterotopia realizado pelo filósofo francês Michael Foucault, para transformar seu reflexo em um dos fenômenos geográficos mais interessantes sobre a construção de um senso de habitar, apropriar e significar territórios e lugares: migração internacional. Neste contexto de compreensão, a construção para os "outros espaços" localizados no que chamamos de espaço real, em alusão ao sociólogo e filósofo francês Henri Lefebvre, permite que este trabalho postule a importância da experiência e da visibilidade das diferenças na compreensão do patrimônio geográfico e cultural dos encontros, isto é como uma forma de observar a riqueza que a pluralidade possui e como uma forma de resistência à estipulação hegemônica antes de compreender a diversidade.

Palavras-chave: Migração internacional, heterotopia, espaço social, patrimônio geográfico, patrimônio cultural.

\section{Heterotopic dimension of international migration: The spatial value of geographical heritage}

\begin{abstract}
The present work takes the concept of heterotopia realized by the French Philosopher Michael Foucault, to turn its reflection towards one of the most interesting geographical phenomena with respect to the construction of sense to inhabit, to appropriate and to mean territories and places: the international migration. In this framework of understanding, the construction towards those "other spaces" located in what we call real space, alluding to the French sociologist and philosopher Henri Lefebvre, his work can reflect on importance of experience and visibility of differences in understanding the geographical and cultural heritage of meetings this as a way to observe the richness that has the plurality and as a form of resistance to the hegemonic stipulated before understanding diversity.
\end{abstract}

Keywords: International migration, heterotopia, social space, geographic heritage, cultural heritage

Recebido: janeiro de 2018. Aceite: março de 2018.

${ }^{1}$ Doutora em Geografia pelo Centro de Investigaciones en Geografía Ambiental, Universidad Nacional Autónoma de México/UNAM e Professora Associada do Departamento de Estudios Culturales, Demográficos y Políticos de la Universidad de Guanajuato, México. Email: miriam.reyes@ugto.mx.

${ }^{2}$ Doctor en Geografía por el Centro de Investigaciones en Geografía Ambiental, Universidad Nacional Autónoma de México/UNAM. Email: idhinojosaf@gmail.com. 


\section{Introducción}

En una conferencia dictada el 14 de marzo de 1967 en el modernista enclave parisino del Circulo de Estudios Arquitectónicos, el filósofo, Michael Foucault, planteó algunos de los contornos y posibilidades de uno de los conceptos que, desde la geografía, permite establecer un acercamiento hacia la complejidad de la experiencia contemporánea del espacio, desde su doble posición como campo generalizador y al mismo tiempo disgregativo, es decir, heterótopico.

La acotación de la heterotopía a los fenómenos geográficos en torno a la movilidad, así como a sus repercusiones; permite reflexionar sobre la construcción de esos "otros espacios" situados en aquello que llamamos espacio real. Es decir, y en palabras más precisas, entender que los procesos de sociabilidad entre actores que migran de un lugar diferente al suyo, como lo es el caso de la migración internacional, conllevan a entender sus espacios de resistencia, como menciona Foucault (s/f) "aquellos lugares que se oponen a todos los demás y que de alguna manera están destinados a borrarlos, compensarlos, neutralizarlos o purificarlos". Y más allá de esto, observar cómo esos "otros espacios" en la vida cotidiana van ofreciendo complejidades para su empoderamiento, o mejor dicho, para su apropiación, uso y significado.

Es por lo establecido anteriormente, que la inscripción de este trabajo tiene el objetivo de establecer el entendimiento de la complejidad y la diversidad resultantes de la creación de espacios "reales o verdaderos" que se legitimizen o empoderen desde el encuentro con lo otro. Dichos elementos, desde el pensamiento de Foucault, permitirían hablar sobre la calidad imaginaria o virtual que localiza a estos espacios fuera de todos los sitios; los cuales, desde la postura del encuentro, crean horizontes de definición y de creación, desde la resistencia al olvido o la disgregación espacial y social. Con la finalidad de retomar la complejidad entre heterotopía y espacio, el artículo principia articulando una aproximación de la heterotopía, su importancia y relación con el ámbito de la migración y la geografía; posteriormente la heterotopía es vinculada a la aportación del patrimonio identitario geográfico en los espacios de resistencia: esos otros espacios, que son interesantes desde su propia conformación, significación y diferenciación, entendidos como espacios de producción de sentido.

\section{La heterotopia en la migración}

El filósofo francés Michael Foucault, en su conferencia "los espacios otros" alude la distinción existente entre la utopía y la heterotopía. La utopía la define como la yuxtaposición en un único lugar, que remite a un lugar universal, pero sin lugar real, y a un futuro distante, construido fuera de nuestro espacio y tiempo, diría Foucault, es un emplazamiento, una irrealidad. Las heterotopías, por otro lado, las sitúa como contraposicionamientos, espacios reales que realizan una utopía; es decir, espacios que se yuxtaponen, que permiten pensar la creación de otros espacios, de nuevos lugares a partir de un cambio de perspectiva. Las heterotopías, a diferencia de la imaginación y su proyección en un horizonte distante -característicos de la utopía-, se convierten en algo que consideramos y valorizamos positivamente, es decir, remite a lo otro a través de la construcción de un mundo "irreal".

La heterotopía, al referir la construcción de lo otro, es el emplazamiento real que se puede encontrar en el interior de la cultura, como define Foucault (1967) es una "[...] especie de lugares que están fuera de todos los lugares, aunque sin embargo efectivamente localizables. Estos lugares, puesto que son absolutamente otros que todos los emplazamientos que reflejan y de los que hablan, los llamaré, por oposición a las utopías, las heterotopías" (3). Estos otros espacios, de manera referencial ante lo otro, nos acotan hacia nuestra propia conducta como individuo y como sociedad a partir de la extrañeza, la diferencia y por supuesto, en la propia coexistencia del ser humano.

Demarcar un sentido geográfico a la propuesta heterótopica de Foucault, nos obliga a precisar en la importancia que posee la vida cotidiana en la construcción del sentido espacial en las actuales sociedades. Como apunta Alonso (2014), actualmente las definiciones y exclusiones de lo propio y lo ajeno se van desdibujando o se superponen ante la tensión. En este sentido, se establecen así múltiples realidades derivadas de la diversidad de escenarios posibles, producto de la yuxtaposición, en donde las relaciones cotidianas más que contraponerse se nutren las unas a las otras, e inclusive, pueden potencializarse, tanto en un aspecto positivo como en uno negativo.

Ante lo anterior, pensemos en el caso de la migración internacional, en donde la legitimidad hacia su visibilidad o invisibilidad atiende a una relación de sentidos otorgada por el sujeto o por fuera de este. En tal hecho, teniendo como elemento principal el control que pueda otorgarse a la estigmatización o construcción de narrativas alrededor de la migración, originando con ello, representaciones geográficas que pueden ser 
señaladas con base en Foucault (1967) como oposiciones espaciales. Es decir, la relativización hacia los espacios públicos y privados, los espacios sociales y los espacios familiares, entre otros.

Estas oposiciones espaciales que después podrán ser yuxtapuestas aluden a las dos acepciones de heterotopía dilucidadas por Foucault, las heterotopías de crisis y las heterotopías de desviación. La primera de ellas, las heterotopia de crisis, refiere los lugares privilegiados, sagrados, o prohibidos, reservados a los individuos que se encuentran, en relación a la sociedad y al medio humano en el interior del cual viven, en estado de crisis. La segunda, las heterotopias de desviación, son aquellas en las que se ubican los individuos cuyo comportamiento está desviado con respecto a la medida o a la norma exigida (Foucault, 1967).

Partiendo de una postura geográfica, las acepciones de heterotopía, tanto la de crisis como de desviación, muestran las tensiones que se convierten en enclaves de procesos de espacialización, cuya concreción es la construcción espacial de los lugares a través de lo que puede ser el uso narrativo de la heterotopía. Prosiguiendo con esta idea, la narrativa permite transformar una heterotopía de crisis en una nueva heterotopía, tal como es visible en los lugares santuario de la migración, particularmente en los Estados Unidos de Norteamérica. En este sentido, los santuarios son lugares que se sobreponen, son establecidos para aislar y unificar; pero que además desde su propia concepción son espacios en constante tensión por demarcar "lo propio" con lo "ajeno", sin saber qué es lo ajeno y qué es lo propio para el habitante. Partiendo de la construcción de cotidianeidad, los santuarios se convierten en enclaves de imaginación para los habitantes.

Los enclaves de la imaginación y su relación con las heterotopías, de acuerdo con la concepción de Lestegás (2006), retoman el fenómeno de la heterogeneidad del espacio, pero considerando además la exclusión como un caso particular de heterotopía:

lugares destinados a acoger la diversidad cultural y social que integra el mundo de la exclusión. Se trata, en suma, de espacios en los que la 'otredad', la alteridady las conductas normativas se pueden explorar sin reducirlas a simples productos imaginativos u oníricos, sino materializándolas y considerándolas en relación con los productos sociales existentes (Lestegás, 2006, p. 178).

De tal forma, estos lugares dotados de "otredad" permiten ahondar en la indagación de la dimensión simbólica de la exclusión. A través de un punto de vista de creaciones narrativas, como apunta Alonso (2014, p. 337), la exclusión sería una suerte de diferenciación espacial, en la cual "la heterotopía es, como todas las diferencias, siempre 'para' algún grupo, siempre en 'relación con', más que un ser 'en sí' ". Razón por la cual, las heterotopías se convierten en lugares conscientemente construidos, pero con una particularidad: se sobreponen a los lugares culturalmente normalizados, o bien, cotidianamente asimilados. En la figura 1 se puede apreciar la sobreposición de una construcción simbólica en un espacio público, descubriendo la esencia de la diferencia.

Pensemos una vez más en el caso de la migración. Hablar de la heterotopía de la migración es dilucidar no sólo la historia de las interrelaciones entre los territorios y sus actores migrantes, es decir, entre el que se va, el que recibe y el que se queda; si no también su construcción histórica, social y cultural. Hablar de la heterotopía de la migración, es hacer alusión a las diferentes prácticas simbólicas y de construcción de significados que se dan por la movilidad, la fijación y, la permanencia e incluso por el olvido. Es pensar, en la vinculación que se establece entre la movilidad y la cotidianeidad a fin de observar los diferentes modos de identificación, pertenencia y apropiación, vía la ritualización o, desde una postura geográfica y cultural, su patrimonialización.

A través de esta idea de heterotopía de la migración, podemos pensar en la complejidad que presenta, a nivel espacial, social y sobretodo, de significado, el transitar entre dos lugares que comparten un territorio y que por su condición de movilidad, la cotidianeidad desde el propio espacio de vida alude a un "dentro de" para reafirmarse y distinguirse.

El pensamiento heterótopico de Foucault nos permite atender el análisis provisto hacia las formas sociales de producir un espacio. En este sentido y desde una similitud con Lefebvre (2013), el espacio se presenta como producto y medio de producción. Con la abstracción del espacio, las formas sociales de producirlo son alineadas y por tanto, modificadas. De acuerdo a Lefebvre (2013), la producción del espacio social se concibe mediante tres aristas: la vida en el espacio, la representación del espacio y el significado del espacio. Esta triada implica, por sí misma, la concepción tanto de un espacio que se vive, como un espacio en el que se vive. Los cuales, forman parte de la traída al representarlos y significarlos. Por otro lado, el conflicto de la heterotopía puede representarse como resultado de la yuxtaposición del espacio abstracto - el espacio representado - y los espacios de representación de quienes viven el lugar. 
Figura 1. Representación heterótopica de la migración mexicana en Chicago.

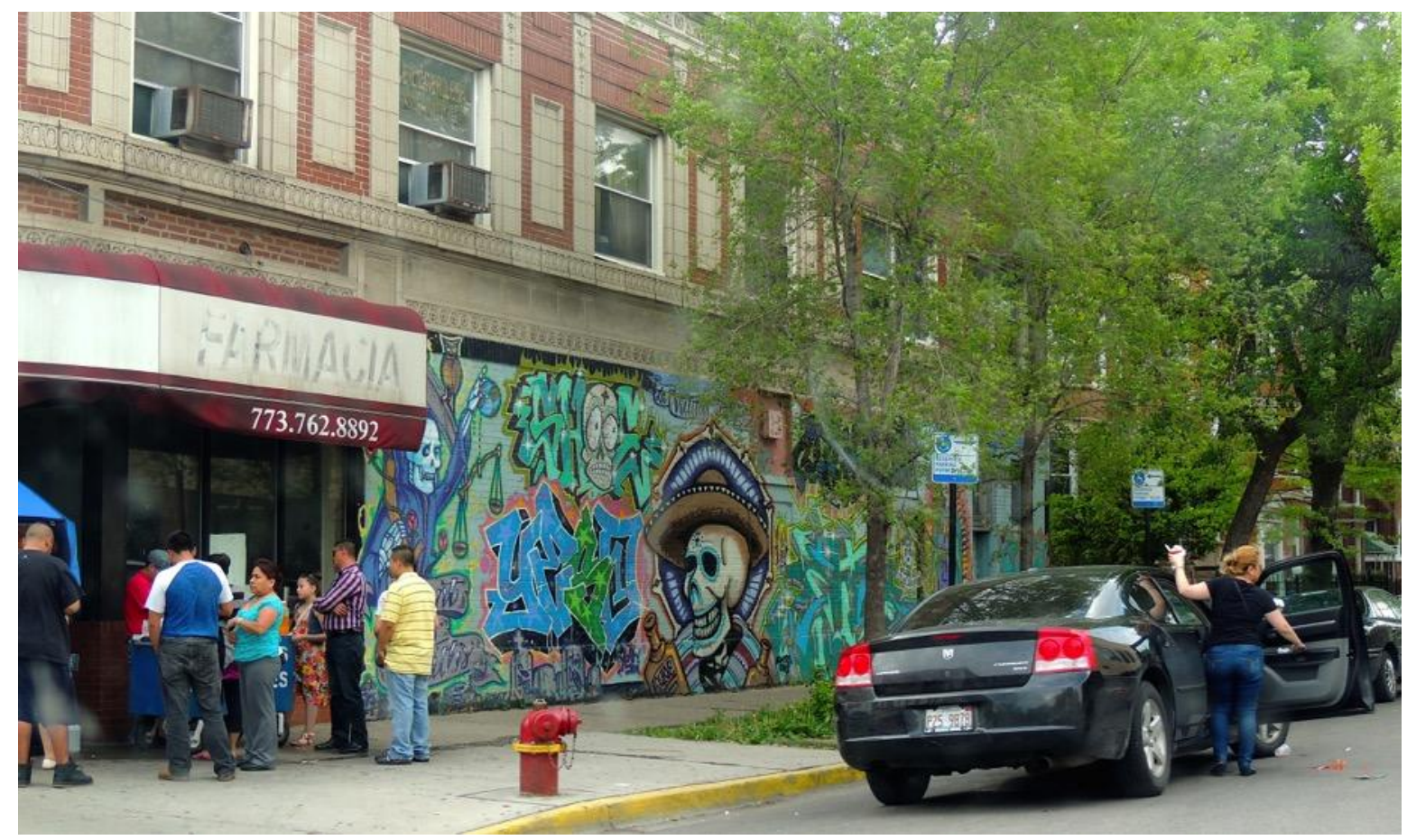

Fuente: Archivo personal. Miriam Reyes Tovar. Mayo, 2015.

\section{La producción simbólica del espacio heterótopico en la migración}

Con el objeto de sustentar una crítica de las heterotopías relacionada con el estudio del espacio social, se propone establecer símiles entre el pensamiento de Foucault y Lefebvre. De esta forma, sostenemos que un espacio heterótopico en la migración, es un espacio representado que irrumpe un espacio real o abstracto. Aunque en el pensamiento de Lefebvre (2013), el espacio abstracto irrumpe en la triada e impone una producción del espacio ajena a la sociedad o comunidad que inicialmente producen. En el pensamiento heterótopico, la yuxtaposición de sentidos permite aludir al campo reflexivo y de expresión de sentido del vivir y experimentar la superposición de lugares. En este caso, el espacio producido es producto de dos momentos claves de la migración: 1) la movilidad causada por la irrupción del espacio abstracto a las comunidades y 2) el espacio abstracto asimilado en los espacios por la representación (principalmente en espacios periféricos) que empujan la migración mediante la fabricación de un utopismo que se materializa en la yuxtaposición producida por la movilidad, alimentada a su vez por el espacio abstracto inicial.
La irrupción ocurre cuando la movilidad, como producto espacial del primer momento, producido en un espacio representado que reconfigura el espacio abstracto mismo, reencuentra el espacio abstracto, pero en una suerte de ensamblaje que materializan la heterotopía en el lugar de destino, producto de los simbolismos de la reconfiguración en el lugar de origen con el encuentro del espacio abstracto en el momento y lugar de destino de la movilidad. Por lo tanto, este espacio representado, producto de un espacio de representación, influye en la forma en la que el espacio se vive y se significa en el lugar y momento de encuentro con el destino de la migración.

Lo anterior y retomando la idea de heterotopía de la migración, puede ejemplificarse en el encuentro entre dos formas de producir el espacio que el migrante experimenta. La primera, en la producción del espacio social establecido en su comunidad de origen, la cual brinda códigos de significados y representaciones en su espacio de vida; y en segundo aspecto, la exposición de dichos códigos en un espacio de vida distinto al suyo. Esta nueva forma de vivir en otro lugar, en consecuencia, alimenta nuevos códigos y significados en su nuevo contexto. Presenciando dos fuerzas principales que modifican una nueva producción del espacio, a partir del conjugar elementos que se llevan o se portan, y que, en un nuevo lugar, se descubren. 
De tal forma, podemos afirmar que la migración tiene un efecto sobre el migrante derivado del encuentro, a partir de la forma de producir el espacio mediante los espacios de representación de la comunidad de origen; y los espacios representados de la sociedad que los recibe. En este sentido, los espacios de representación continúan vigentes en Estados Unidos en términos de relación con la comunidad de origen. Sin embargo, la producción del espacio social, basada en los espacios de representación comunitarios en Estados Unidos, son permisibles por los espacios representados que se encuentran en las sociedades de arribo, tal como lo expuesto con los lugares santuarios

Estas fuerzas tienen diferentes temporalidades que son de alguna forma vinculadas por la migración. Por un lado, el papel de la comunidad de origen que se deja y sus propias transformaciones dadas en el tiempo; y por lo tanto, en el espacio que hereda a las instituciones que lleva consigo el migrante. Aunque quizás llevados con él hacia otro lugar con un ritmo diferente. Por otro lado, el de la sociedad que lo recibe en un espacio representado donde es esperado y asignado como un elemento asimilado a la fuerza de trabajo; sin embargo, estos espacios representados han sido también espacios en los que la comunidad ha logrado surgir a través de la extensión de los lazos comunitarios que han producido un espacio de representación al interior de los espacios representados.

Esta disgregación del tiempo y localización en otro lugar diferente, como lo es la extensión dada por la migración, deja ver el proceso de heterocronía propuesto por Foucault (1967), el cual es asociado con cortes de tiempo como forma de ruptura con el tiempo tradicional. A partir de una acepción netamente geográfica, la heterocronía puede incidir en la construcción narrativa de un imaginario geográfico que pone en juego la idealización o incluso, un "estancamiento de tiempo" ante la nostalgia.

En este sentido, y como una forma de establecer una acotación heterótopica concerniente a la creación de "los otros espacios" en función de imaginario geográfico, la heterocronía proyecta modos de concebir y practicar el espacio, como el resultado de la relación entre discursos, fantasías y corporalidad situados entre el yo y la otredad, entre lo que es distinto a mi y lo que yo no represento, entre lo que conforma mi espacio, con aquello que me lo confronta (Rose, 1999; Said, 2008).

Esta forma de concebir el espacio, en tanto campo de experiencia sensible y de creación imaginativa, permite atender su ámbito reflexivo, como constructo de una "imaginación geográfica" (Soja, 2010) y de una vivencialidad por parte del sujeto (Kant, 1984). Con la idea de "la imaginación geográfica" en esta acotación heterotópica de la migración, hacemos mención, al establecimiento de los imaginarios de la movilidad, en termino de imaginarios geográficos que conllevan una fuerte carga social, y que, a su vez, crean complejidades en la vida cotidiana de los sujetos.

Los imaginarios participan en la construcción, moldeado, negociación e inclusive perdida de los lugares (en un sentido creativo); crean relaciones entre lo familiar y lo desconocido. Los imaginarios aquí, desde el punto de vista de la heterotopía de Foucault, se nos muestran como el cumulo de lugares que pueden crear y legitimar un nuevo espacio.

Se crean antagonistas, protagonistas, héroes, culpables, que van creando nuestra actualidad, y que cada uno de ellos busca empoderarse de su espacio y dar legitimidad a ello. Los imaginarios entablan diferencia con el pasado y se vinculan con el presente; los imaginarios "heterotópicos" muestran una diversidad, se nos presentan como "espacios contrarios", como productores de experiencias mixtas entre realidades y mitos paradojales; los imaginarios geográficos de la migración son espacios heterotópicos de crisis que crean inestabilidades y contrastes.

Los lugares santuarios de los migrantes latinos en Estados Unidos, las zonas urbanas de los llamados cinturones de miseria o asentamientos de migrantes lejos de los centros urbanos, las zonas de refugiados en Europa, las zonas de frontera entre países que llevan consigo personas que deambulan entre el ser y la nada, entre el quedarse o morir; los turistas que se apropian de las ciudades, al menos en sus imaginarios por un instante, son ejemplos de cómo los sujetos se relacionan con sus espacios a nivel de prácticas sociales y culturales que después marcarán la pauta del ser.

Las asociaciones de lugares van conformando mapas imaginarios de vigencia y proyección en modos de concebir y practicar la cotidianeidad. Los cuales, en muchas ocasiones estructuran y alimentan las discriminaciones y prejuicios entre lo que es y lo que debe de ser. Observar la dimensión simbólica del espacio, a nivel de campos que se crean y alimenta de vivencialidades e imaginarios, es mostrar cómo las heterotopías pueden ser las distintas formas de resignificación y reapropiación que garantizan la supervivencia de las personas que vivencian el espacio, con todas sus contradicciones y conflictos de interés.

De tal forma, la construcción de sentido heterótopico, nos permitimos llamar así a la forma sensible de la coexistencia de sistemas simbólicos, impactan en el espacio de la vida de los sujetos a través de la constante yuxtaposición de 
singularidades como los cierres y las aperturas, como las ilusiones y las realidades (Posada, 2014). Pero sobre todo, por los conflictos. Es por ello que hablar de una producción del espacio, en términos de heteropía, a nivel de producción simbólica y de crisis, nos lleva a la percepción de la experiencia del espacio, y al significado de la experiencia.

Lefebvre (2013) destaca, desde el espacio percibido, el espacio concebido y el espacio vivido, los ejes de traducción para estructurar el espacio social partiendo del mundo físico y la experiencia en él mediante la práctica, seguido de la percepción y finalmente, el espacio pensado, concebido o el significado de la experiencia, tal como puede ser visto en la figura 2. Sin embargo, los espacios de representación de un lugar, donde la triada espacial es reproducida; pueden ser suplantados por un sistema totalizador, o en un espacio complejo, en el cual los espacios de representación de las comunidades conforman un espacio representado, producido desde el exterior. sombra que me da a mí misma mi propia visibilidad, que permite mirarme allá donde no estoy" (Swain, 2001, p. 340), pero que también puede colapsar, cuestionar e inclusive mirarse desde lo que ya no soy, pero que me vuelve a posicionar ante lo que fui,

En segundo lugar, su patrimonalización, en la cual, hacemos alusión a las distintas formas que se tienen por dar legitimidad a la diferencia, por recrear y crear desde la contradicción, o propiamente dicho, desde la resistencia al olvido y a la invisibilizarían. La importancia que posee el patrimonio identitario geográfico desde su propia construcción como campo heterótopico de la migración, permite atender no sólo la movilidad y las diferentes prácticas espaciales que de ésta se derivan, sino también cómo la distancia y la proximidad, lo cercano y lo lejano, lo inmediato, lo pasado y lo futuro, dan cuenta de relaciones de poder, de relaciones socioespaciales e identitarias.

Figura 2. Representación heterótopica de la migración mexicana en Chicago.

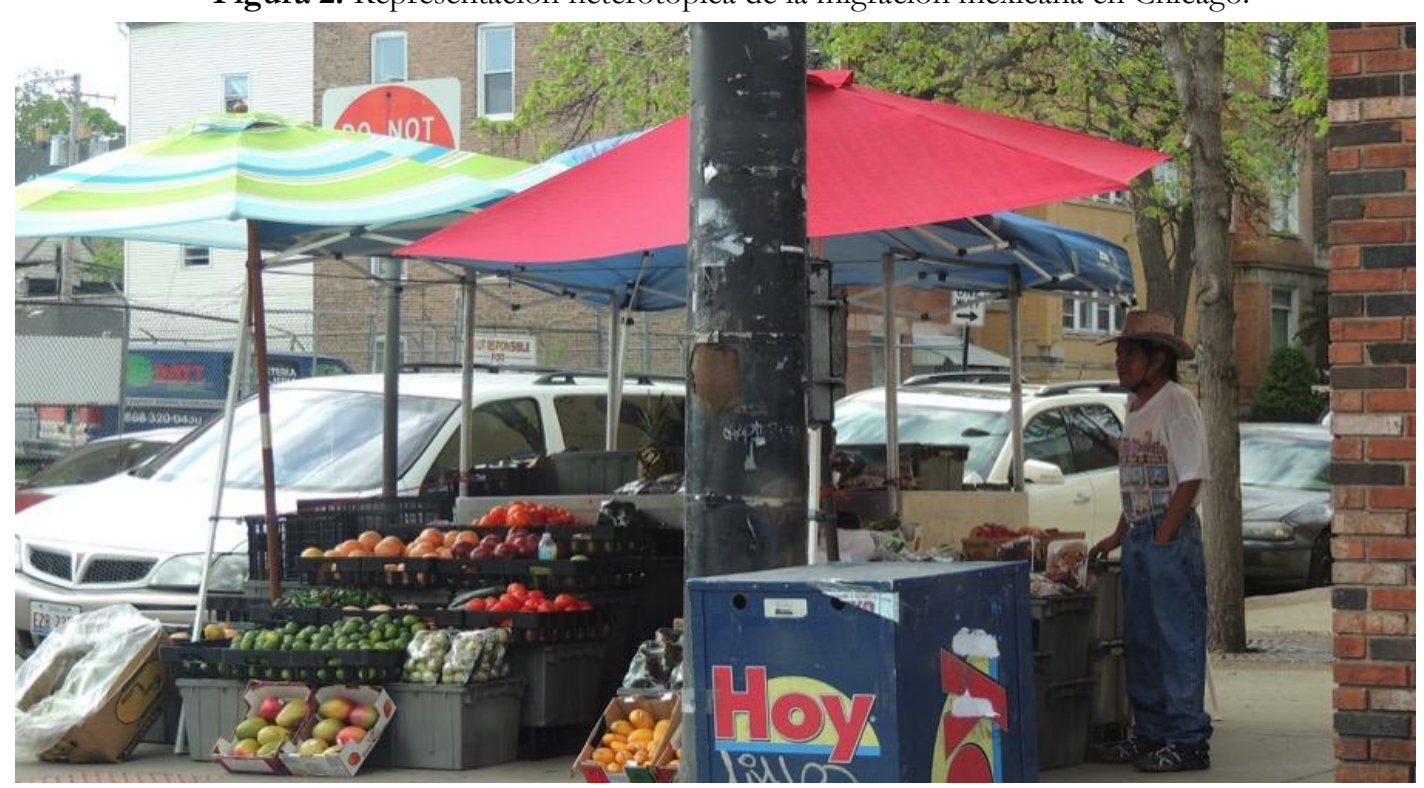

Fuente: Archivo personal. Miriam Reyes Tovar. Mayo, 2015.

Así, la acotación heterótopica en y de la migración, versa, desde nuestro punto de vista, particularmente en dos ámbitos que son de vital importancia al hablar de los sujetos en su relación con la vida cotidiana y esta idea de "apropiación y significación de los espacios", tanto para su uso como para su legitimidad:

El primero de ellos es una "heterotopía identitaria", es decir, el entendimiento de mi "yo" como un espacio que se crea, recrea, contradice e inclusive se transiciona. Swain (2001) menciona "yo soy el espejo de mí, un lugar sin lugar en un espacio irreal que se abre virtualmente atrás de la superficie, yo estoy allá donde no estoy, una especie de
Esta reflexión ha dado paso a la construcción de discursos y lenguajes propios respecto al papel del imaginario y lo simbólico en dicha relación. Se ha dado paso a una experiencia sensible y vivencial del espacio como experiencia, como elemento constructor y significador de la vida del sujeto en su lugar.

En la relación espacio - experiencia sujeto, desde el punto de vista de la geografía humana, el discurso hacia la centralidad del sujeto, a través de las distintas formas que tiene por hacerse en y con el espacio; observar su capacidad de "habitar", con base en Hiernaux (2008), se delinea la forma en la cual las prácticas hechas por 
las personas permiten acceder a un nivel analítico que aborda la construcción social del territorio, particularmente, desde la significación que un determinado espacio de vida tiene para los sujetos.

De tal forma, observar al territorio como una visión del mundo por parte de sus actores, permite en primer lugar hablar de su existencia y sus experiencias vividas en los lugares; y segundo, crear una suerte de tensión dinámica entre lo habitual, la rutina, la reproducción, la creatividad, la innovación, el cambio y la espontaneidad adaptativa (Claval, 1999; Lévy \& Lussault, 2007). En este sentido, la migración internacional, posee una fuerte carga territorial que permite marcar el contexto no sólo espacial, sino también temporal y cultural de la forma en la cual los sujetos establecen narrativas de las formas de habitar y significar los territorios, y sobretodo, siguiendo la línea de pensamiento heterótopico, el poder yuxtaponer lugares significativos e incluso transgredirlos vía la resistencia del recuerdo y la memoria.

\section{E1 patrimonio geográfico desde el acotamiento heterótopico de la migración}

Posicionar a los territorios identitarios que son caracterizados por las vivencias y trayectorias cotidianas que establecen el sentimiento de pertenencia y formación de identidades individuales, permite hablar de una conciencia espacial compartida, resultante del entramado de relaciones simbólicas en el establecimiento de una "identidad del territorio" y de una "identidad para el territorio" (Guérin-Pace \& Guermond, 2006)

Guérin-Pace (2006; 2003) señala que existe un sentimiento de pertenencia a un territorio, visto como un componente espacial en la construcción identitaria de los sujetos, el cual se crea mediante el ensamble de cursos geográficos y significados otorgados a los lugares inscritos sobre el territorio por parte de los sujetos. En la pertenencia territorial, la autora señala que la formación de un patrimonio identitario geográfico puede ser movilizado por los individuos, ya que al tener como centro de referencia al territorio, en él se establecen y reconocen vínculos de pertenencia (Ortega, 2007) que se fijan pero que pueden ser dinámicos.

En el dinamismo de los vínculos con el territorio, la relación identidad y territorio en la migración, con base en Guérin-Pace y Guermond (2006) permite atender la identidad del territorio, como la puesta en escena de los datos específicos de una entidad geográfica, resaltando su ubicación, patrimonio y características culturales de sus habitantes; por su parte, hablar de una identidad para el territorio, es hacer alusión al carácter geográfico de las identidades individuales construidas en el territorio (Guérin-Pace \& Guermond, 2006, p. 289); y al establecer la relación entre lo individual y colectivo dentro de sus contextos territoriales específicos se establece una conciencia espacial compartida (Mazurek, 2009).

La identificación hacia un territorio estará en función de las diversas formas de objetivación del mismo, es decir, a partir de tomar a la historia, cultura, símbolos y prácticas socio-culturales adscritas al territorio (Gendreau \& Giménez, 2002), se establece una apropiación territorial, afectiva y simbólica por parte de los sujetos hacia un determinado territorio, teniendo una inscripción o pertenencia hacia el lugar; de tal forma, la comprensión espacial de pertenencia a ese lugar, da muestra del patrimonio geográfico, como una forma de identidad colectiva que supone al mismo tiempo, una adhesión que puede ser compartida y entendida como una "identidad hacia una entidad geográfica" (Guérin-Pace \& Guermond, 2006, p. 298). Pero, que, desde el punto de vista de la migración, los anclajes de pertenencia e identificación socio-espacial, remiten a una forma cambiante, reconfigurada o traducida de la forma en la cual la pertinencia territorial establece una identificación, apropiación y comprensión espacial de su habitar (Guérin-Pace, 2006, p. 102).

En este sentido, y desde un discurso heterótopico de la migración, el patrimonio geográfico se vuelca hacia un cumulo de significados que se nutren del patrimonio cultural, entendiendo por este una expresión simbólica. Con base en Batalla (1991, p. 119), "[...] el patrimonio cultural abarcaría también costumbres, conocimientos, sistemas de significados, habilidades y formas de expresión simbólica que corresponden a esferas diferentes de la cultura".

Abordar la noción de identidad colectiva de una comunidad a través del reconocimiento no sólo de sus miembros entre sí, sino también de sus elementos culturales, como lo es la propuesta de Batalla respecto a la noción de patrimonio cultural, en un ámbito de movilidad, como lo es la migración, nos lleva al laberinto de significados que se crean con y en la estructuración de la identidad simbólica del sujeto y de la colectividad, estableciendo una suerte de universos simbólicos que se superponen los unos a los otros.

En este sentido, el patrimonio geográfico de la migración, desde su arista cultural y de yuxtaposiciones, nos permite entenderlas como elementos de anclajes simbólicos y significativos que les otorgan su encuentro con la tradición y la modernidad, con base en Di Meo (1996, p. 93):

El universo simbólico (ideologías territoriales, valores patrimoniales, memoria colectiva, sentimientos identitarios 
en particular) de las estructuras cognitivas del sujeto social encuentran un campo de referencias solidas sobre las estructuras objetivas del espacio geográfico. Estas aportan los argumentos identitarios, de innumerables marcas susceptibles de revitalizar la memoria colectiva por las sociedades que las producen.

En este sentido, la dimensión simbólica del espacio, o del patrimonio geográfico y cultural, estarán dados respecto a la construcción e identificación territorial ligada a la visión del mundo de cualquier grupo o individuo, permitiendo observar las estructuras hechas por el sujeto sobre el territorio como una forma de reconocimiento, identificación y pertenencia. Ante lo anterior, nuestro discurso y posicionamiento nos lleva a mostrar a los espacios heterótopico de la migración, en los espacios de representación.

Figura 3. Representación heterótopica de la migración mexicana en Chicago.

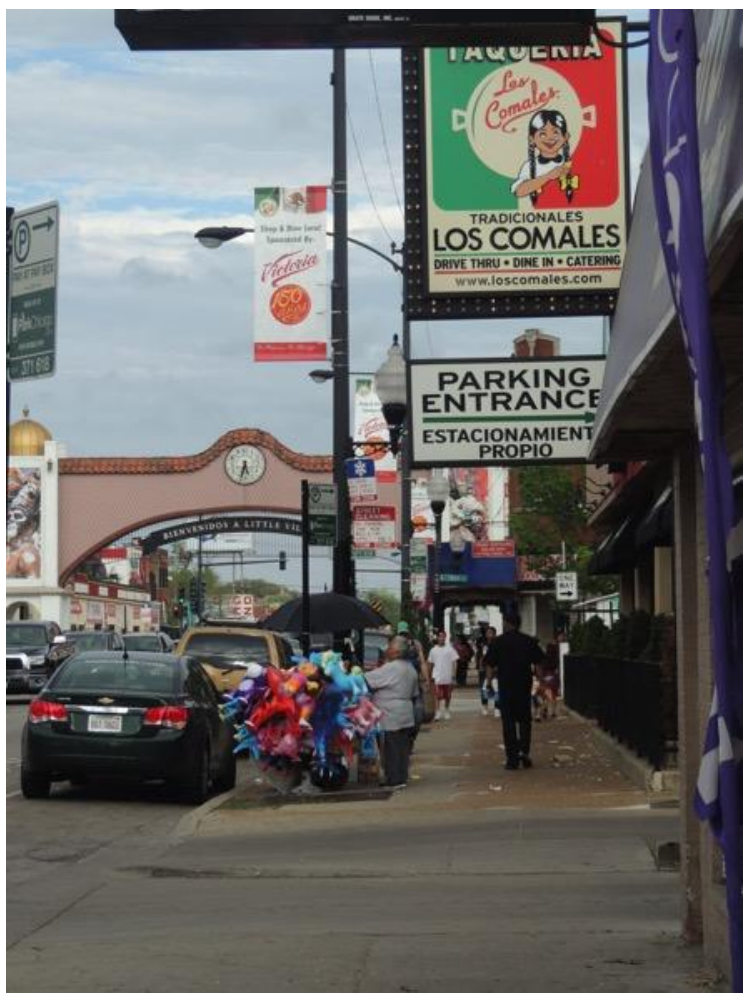

Fuente: Archivo personal. Miriam Reyes Tovar. Mayo, 2015.

En el caso particular de la migración, los espacios de representación tuvieron que sobrevivir clandestinamente en un principio, para luego ser asimilados por los espacios de representación y finalmente tolerados $\mathrm{y}$ encapsulados en lugares determinados por la representación, tal como puede apreciarse en la figura 3 , donde la espacialización de la mexicanidad ha sido sobrepuesta al espacio norteamericano, convirtiéndose así, en un espacio de representación que fue integrado $y$ principalmente, tolerado como espacio representado de la diferencia étnica y racial.

No obstante, la tolerancia vía integración, es cambiante y muestra la fragilidad en términos legales de los migrantes, aunque también, evidencia la fortaleza de una comunidad que ha logrado abrir espacios de legitimidad y permanencia. Con base en esta idea, podemos afirmar que la creación de los espacios de representación, o lo que hemos tratado de dilucidar como espacios heterótopico, ha sido posible en un país donde la dualidad integración disociación permite la existencia del encapsulamiento de espacios de representación en una amalgama de espacios representados como una unidad nacional.

\section{Conclusión}

Observar al patrimonio como una forma en la que se conjunta un tiempo pasado con un presente y cuyo marco de acción es el territorio, es conferirle un valor patrimonial al territorio (Di Méo, 1996); donde la dupla patrimonio y territorio establecen la aparición de un espacio común, un espacio en el cual los sujetos se reconocen y se crean, estableciendo una "transferencia de sacralidad de lo social a lo espacial” (Di Méo, 1996).

La yuxtaposición de espacios que la migración origina, en alusión a Lefebvre (1983), es trascendental, principalmente para entender las transformaciones que a nivel territorial sucede, y sobretodo en tratar de visibilizar, los cambios que se producen en el imaginario global de lo qué es una sociedad.

En el caso de la migración, los espacios heterotópicos que pueden producirse por el contacto con lo diferente, el recuerdo de lo dejado y el sueño de lo que puede encontrarse o materializarse, establecen como menciona Harvey (2017) relaciones potencialmente transformadoras con los demás espacios. El problema, prosiguiendo con Harvey, radica en encontrar formas de hacer realidad sus potencialidades efímeras (Harvey, 2017, p. 187).

La importancia de retomar una reflexión hacia el pensamiento de Foucault y Lefebvre en las particularidades territoriales que tiene la migración, postulamos, se encuentra en hacer notar cómo el patrimonio territorial hace alusión a un encuentro que permita observar las diferencias, pero también las nuevas creaciones. Nos invita a observar cómo el patrimonio identitario de la migración, desde una acotación heterótopica, puede revertir la idea de Foucault respecto a la forma en la cual, "el emplazamiento sustituye al entendimiento que, a su 
vez, reemplazaba la localización" (Foucault, 1967), si bien, la localización puede ser lejana y cercana, la vivencialidad y las diversas formas de expresarlo, pueden establecer un valor espacial del patrimonio geográfico de la movilidad.

\section{Referências bibliográficas}

Alonso, M. G. (2014). Los territorios de los otros: memoria y heterotopía. Cuicuilco, 21(61), pp. 333-352. Recuperado de http://www.scielo.org.mx/scielo.php?script =sci_arttext\&pid=S0185$\underline{16592014000300015}$

Batalla, G. B. (1991). Pensar nuestra cultura. Ensayos. México: Editorial Patria.

Claval, P. (1999). Los fundamentos actuales de la geografía cultural. Documents d'Análisi Geográfica. 34, pp. 25 - 40. Recuperado de: http://age.ieg.csic.es/hispengeo/docum entos/clavalcultural.pdf

Di Méo, G. (1996). Les territoires du quotidien. Paris: L'Harmattan.

Foucault, M. (1967). De los espacios otros. Conferencia dictada en el Cercle des études architecturals. Recuperado de: http://yoochel.org/wpcontent/uploads/2011/03/foucalt de-losespacios-otros.pdf

Gendreau M., \& Giménez, G. (2002). La migración internacional desde una perspectiva sociocultural: estudio en comunidades tradicionales del centro de México. Migraciones Internacionales, 1 (2), pp. 147-180. Recuperado de http://www.scielo.org.mx/pdf/migra/ $\mathrm{v} 1 \mathrm{n} 2 / \mathrm{v} 1 \mathrm{n} 2 \mathrm{a} 6 . \mathrm{pdf}$

Guérin-Pace, F. (2003). Vers une typologie des territoires urbains de proximité. L'Esapce Géographique, 4 (32), pp. 333-344. doi: $10.3917 /$ eg.324.0333

Guérin-Pace, F. (2006). Sentiment d'appartenance et territoires identitaires. L'Espace Géographique. 4 (35), pp. 298-308. doi: 10.3917/eg.354.0298

Guérin-Pace, F. \& Guermond Y. (2006). Identité rapport au territoire. L'Espace Géographique, 4 (35). 289-290. doi: 10.3917/eg.354.0289

Harvey. D. (2017). El cosmopolitismo y las geografias de la libertad. España: Ediciones Akal.

Hiernaux, D. (2008). De los imaginarios a las prácticas urbanas: construyendo la ciudad de mañana. Iztapalapa - Revista de Ciencias Sociales y Humanidades, (64-65), pp. 17-38. Recuperado de bttp://132.248.9.34/ hevila/Izt apalapa/2008/vol29/no64-65/1.pdf

Kant, E. (1984). Crítica de la razón pura. Madrid:
Alfaguara.

Lefebvre, H. (2013). La producción del espacio. España: Capitán Swiny.

Lefebvre, H. (1983). La presencia y la ausencia: contribución a la teoría de las representaciones. México: Fondo de Cultura Económica.

Lestegás, F. R. (2006). La estrategia socioespacial de las heterotopías: ¿el poder organiza espacios de exclusión o de fijación?. Xeográfica, Revista de Xeografia, Territorio e Medio Ambiente, 6, pp. 171-179.

Lévy J. \& Lussault, M. (2007). Logique de l'Espace, esprit des lieux: Géographies à Cerisy. Paris: Belin.

Mazurek, H. (2009). Migraciones y dinámicas territoriales. In C. Salazar (Ed.), Migraciones contemporáneas: contribución al debate (pp. 1133). Bolivia: Edit. CIDES-UMSA

Ortega, M. J. Q. (2007). Migración, arraigo y apropiación del espacio en la recomposición de identidades socioterritoriales. Cultura y representaciones sociales. Un espacio para el diálogo interdisciplinario. 2 (3), pp. 3567. Recuperado de http://www.journals.una m.mx/index.php/crs/article/view/16252

Morales, J. E. P. (2014). El gobierno urbano: indagaciones alrededor de las heterotopías innovadoras. Caso Medellín. Cuaderno de vivienda y urbanismo. 7 (13), pp. 116-125. Recuperado de http://revistas.javeriana.edu. co/index.php/cvyu/article/view/8753/807 $\underline{2}$

Rose, G. (1999). Performing Spaces. In J. Allen, J., D. Massey \& P. Sarre (Eds.). Human geography today (pp. 247-259). Cambridge, UK: Polity Press.

Said, W. E. (2008). Orientalismo. España: Random House Mondadori.

Soja, E., Benach, N. \& Albet, A. (2010). Edward W. Soja. La perspectiva postmoderna de un geógrafo radical. Barcelona: Icaria.

Swain, T. N. (2001). ¿Qué es el lesbianismo?. São Paulo: Brasiliense.

\section{Cómo citar este artículo}

Tovar, M. R. \& Flores, I. D. H. (2018). Acotación heterotópica de la migración internacional: el valor espacial del patrimonio geográfico. PatryTer, (1), 1, pp. 13-21.

DOI: https://doi.org/10.26512/patryter.v1i1.7112 